\title{
The lift of a cylinder executing rotary motions in a uniform flow
}

\author{
By P. T. TOKUMARU AND P. E. DIMOTAKIS \\ Graduate Aeronautical Laboratories, California Institute of Technology 301-46, Pasadena, \\ CA 91125, USA
}

(Received 30 April 1992 and in revised form 25 February 1993)

The mean lift coefficient of a circular cylinder executing rotary motions in a uniform flow is investigated. These motions include steady rotation, and rotary oscillations with a net rotation rate. Results for the steadily rotating cylinder show that for a given rotation rate, larger cylinder aspect ratios yield higher lift coefficients. It was also found that the addition of forced rotary oscillations to the steady rotation of the cylinder increases the lift coefficient in the cases where the wake would normally be separated in the steadily rotating case, but decreases it otherwise. In addition, a method for estimating the mean lift of a rotating cylinder is presented. Estimates based on this method compare favourably with similar data published for steadily rotating cylinders.

\section{Introduction}

A rotating cylinder moving in a uniform stream experiences a force normal to the direction of motion. Goldstein (1938) refers to several historical papers on both rotating spheres and cylinders, crediting Magnus (1853) with the first laboratory experiments examining the lift on a rotating cylinder. Early in this century, experiments on a circular cylinder rotating about its axis in a uniform flow were performed by Reid (1924), Prandtl (1925), and Thom (1926, 1931), for example. They found that the mean lift of a cylinder was an (unspecified) function of its rotation rate. Their measurements were for Reynolds numbers in the range

$$
4 \times 10^{4}<R e \equiv 2 a U_{\infty} / \nu<1.2 \times 10^{5},
$$

where $a$ is the cylinder radius, $U_{\infty}$ is the free-stream velocity, and $v$ is the kinematic viscosity. More recently, experiments and simulations have been performed at low Reynolds numbers by Badr \& Dennis (1989), Ingham \& Tang (1990), and Tang \& Ingham (1991) for the steady flow past a rotating cylinder, and at higher Reynolds numbers of $10^{3}$ and $10^{4}$ by Badr et al. (1990) for the flow past a cylinder impulsively started in both rotation and translation. Of particular interest is the observation by Badr et al. (1990), that there is no periodic vortex shedding from a cylinder that is rotating with a surface velocity greater than two or three times the free-stream velocity.

As is well known, the mean lift coefficient of a cylinder can be written as

$$
C_{L} \equiv L / \rho U_{\infty}^{2} a=-\Gamma / U_{\infty} a,
$$

where $\rho$ is the fluid density, $L$ is the lift per unit span, and $\Gamma$ is the mean circulation taken around a contour enclosing the lifting body. See for example Taylor (1925), Thwaites (1960). That this circulation could be measured around contours close to the cylinder was shown experimentally by Thom (1931). 
In the potential flow prototype of a cylinder with lift in a uniform free stream, the rotation and lift of the cylinder is modelled by a point vortex of strength $\Gamma$ at its centre. See for example Milne-Thomson (1968, pp. 187-190). When modelling a flow, $\Gamma$ should be considered as a parameter to be adjusted so that the flow is properly represented; e.g., to satisfy the Kutta condition when modelling flow past an airfoil. Proposing that $C_{L}$ can be made arbitrarily large by arbitrarily increasing $\Gamma$ is not helpful; $\Gamma$ is the mean lift (cf. (2)).

Prandtl (1925) argued that the maximum circulation, $\Gamma_{m a x}$, which could be realized about the rotating cylinder was equal to the circulation at which the upstream and downstream stagnation points join on the bottom of the cylinder. From the potential flow analogue of that case, that corresponds to a value of

$$
\Gamma_{\max }=4 \pi U_{\infty} a .
$$

See also the discussion in Goldstein (1938, pp. 81-84). Beyond this point, Prandtl argued that no circulation could be shed by the cylinder to infinity, as it is ramped up from rest to a constant rotation rate, fixing the total circulation at infinity, and the lift of the rotating cylinder thereby. If this argument were correct, the maximum steadystate lift coefficient that can realized would be given by,

$$
C_{L_{\max }}=4 \pi \approx 12.6 .
$$

Note that Prandtl relates the real and potential flow case of the rotating cylinder not by matching the circulation calculated by taking a contour around the cylinder at the surface, as one would expect, but by matching the peak circumferential velocities in the two cases.

The present experiments examine the effect of cylinder rotating on the flow ahead of the cylinder. In order to assess the effects of forcing, the flow ahead of the cylinder was described in terms of the strength and position of a virtual vortex. The strength of the virtual vortex was then related to the lift coefficient of the cylinder. Results obtained for a steadily rotating cylinder (no forced oscillations) compare favourably with similar data published in the literature. It was also found that the addition of forced rotary oscillations to the steady rotation of the cylinder helped increase the lift coefficient in cases where the wake would normally be separated in the steadily rotating case, and decrease it otherwise. It is believed that this is the first investigation of the flow past a cylinder with both net rotation and forced oscillations.

\section{Experimental set-up}

The present experiments were performed in GALCIT 20 in. $\times 20$ in. Free Surface Water Tunnel $\dagger$ (see Ward 1976). A machined Plexiglas cylinder, 1 in. in diameter and an aspect ratio of 18.7 was supported 10 in. above the bottom of the water channel, between 0.5 in. thick Plexiglas fairings placed flush to the sidewalls of the channel. Power was transferred from the motor to the cylinder using steel reinforced neoprene timing belts. These were entirely enclosed in the fairings and did not interfere with the flow. The cylinder was driven by a high-performance DC motor from PMI Motion Technologies (JR16M4CH Servodisk ${ }^{(m)}$ ), capable of tracking an arbitrary command signal within the bandwidth and slewing rates that were investigated. The command

$\dagger$ The set-up of the present experiments is similar to that used in Tokumaru \& Dimotakis (1991), and Tokumaru (1991). 
signal for the cylinder angular velocity was generated by a computer-controlled function generator. The angular position of the cylinder was measured using a BEI Motion Systems Co. 13-bit absolute position encoder connected to the motor driveshaft.

Velocities were measured using a two-channel $(u, v)$ laser Doppler velocimeter. The optics, traverse and analog/digital LDV processor, outlined below, are as employed by Lang (1985). The $u$-channel was only used to measure the free-stream velocity. The $v$ channel velocities were used to estimate the lift coefficient. A Lexel Model 85 Argonion laser operating in single-line mode $(514.5 \mathrm{~nm})$ at about $200 \mathrm{~mW}$ was used for the LDV. The laser beam for the LDV velocity measurements was split into four beams of nearly equal intensity using two cube beam splitters in series, at $+45^{\circ}$ and $-45^{\circ}$, respectively. The beams for the $v$-channel were then Bragg-cell frequency-shifted to accommodate flow reversals. The Bragg-cell frequency offset between the two beams was $300 \mathrm{kHz}$ for the present data. For alignment, to ensure that the beams intersected in a single probe volume, each of the four beams was passed through wedge prisms. An achromat was used to focus the beams in a focal (measurement) volume located in the midspan plane of the FSWT. Light scattered from the focal volume was gathered by two achromats, in series, and focused on a photodiode with an integral low-noise pre-amplifier. The signal from the pre-amplifier was then band-pass filtered above and below the frequency corresponding to the mean flow velocity to isolate the $u$-velocity, and around the Bragg offset to isolate the $v$-velocity. The band-pass ranges were chosen to pass instantaneous velocity fluctuations and were non-overlapping.

For the data in figures 2-4, the signals from the band-pass filters were processed by an LDV signal processor (Lang 1985) and the velocity data were stored on disk. Each data point in figure 2 is an average over about 10000 (instantaneous) velocity measurements. For the data in figure 6, a pair of tracking phase-locked loops were used to lock TTL square waves to the dominant $u$-and $v$-channel frequencies from the bandpass filters. The TTL signals were then read by a (multiple) counter-timer board on a data acquisition computer and stored on disk for later processing.

Flow visualization was accomplished by introducing red dye into the flow ahead of the cylinder through a hypodermic tube. A blue filter was used to darken the red dye marker relative to the white background. Photographs were taken using a $35 \mathrm{~mm}$ camera. Illumination was provided from behind a large (backlit) white sheet of paper suspended, outside the water tunnel, behind the model.

\section{Cylinder motion}

The angular motion of the cylinder in the present experiments can be described by a single equation; i.e.

where

$$
\begin{gathered}
\Omega=\Omega_{0}+\Omega_{1} \sin (2 \pi f t), \\
\Omega \equiv \dot{\theta} a / U_{\infty}
\end{gathered}
$$

is the normalized rotation rate of the cylinder, $a$ is the cylinder radius, $U_{\infty}$ is the freestream velocity, $t$ is the time, $\dot{\theta}$ is the angular velocity of the cylinder, $f$ is the forcing frequency, and, $\Omega_{0}$ and $\Omega_{1}$ are amplitudes of the steady and harmonic components of the cylinder motion. The normalized forcing frequency is

$$
S_{f} \equiv 2 a f / U_{\infty},
$$

i.e. the forcing Strouhal number. The Reynolds numbers in the present experiments are $R e=3.8 \times 10^{3}$ and $6.8 \times 10^{3}$. 


\section{Lift estimation by the virtual vortex method}

The cylinder in the present experiments experiences no net rotational acceleration after its initial start-up. Hence, once steady-state flow has been established, it cannot shed any additional vorticity into the wake, in the mean. It follows that, some time after start-up, the circulation in the wake cannot contribute to the mean circulation, $\Gamma$, taken around the cylinder. As a consequence, the vorticity contributing to $\Gamma$ must be restricted to a relatively small neighbourhood around the cylinder. This suggests that the effect on the flow, some distance from the lifting body, can be approximated, in a multipole expansion sense, by a single virtual vortex of strength $\Gamma$.

Ideally, in an infinite domain, the transverse velocity $v(x, y=0)$ a distance $x$ upstream of a vortex centre is

$$
v(x, y=0)=-\Gamma / 2 \pi x .
$$

Using (2) and (8), $v$ can be written in terms of the lift coefficient, $C_{L}$. For the infinitedomain case this can be written as

$$
\frac{v(x, y=0)}{U_{\infty}}=\frac{C_{L}}{2 \pi} \frac{x}{a} .
$$

Because of the finite height of the test section, however, a proper account should include an infinite series of spatially periodic image vortices located above and below the cylinder. See figure 1. In this way, symmetry guarantees a no-throughflow condition at the top and bottom of the test section. An expression for an infinite series of vortices, of the same sign, spaced horizontally (instead of vertically), can be found, for example, in White (1979, pp. 470-471). Two such expressions, one for the positivesigned vortices and one for the negative, are superimposed, a half a period out of phase and then evaluated along the $x$-axis to yield the following equation, which replaces (8):

$$
v(x, y=0)=-\frac{\Gamma}{2 h} \operatorname{cosech}\left(\pi \frac{x-x_{0}}{h}\right),
$$

where $h$ is the test section height and $x_{0}$ is the streamwise position of the virtual vortex.

Rearranging (10) yields

$$
\frac{2 h v(x, y=0)}{\Gamma}=-\operatorname{cosech}\left(\pi \frac{x-x_{0}}{h}\right),
$$

or, using (2),

$$
\frac{v(x, y=0)}{U_{\infty}}=\frac{a}{2 h} C_{L} \operatorname{cosech}\left(\pi \frac{x-x_{0}}{h}\right) .
$$

A more general form of (12) which allows for both a streamwise $\left(x_{0}\right)$ and transverse $\left(y_{0}\right)$ displacement of the virtual vortex is

$$
\frac{v(x, y=0)}{U_{\infty}}=\frac{a}{2 h} C_{L}\left[\frac{\cos \left(\pi y_{0} / h\right) \sinh \left(\pi\left(x-x_{0}\right) / h\right)}{\sin ^{2}\left(\pi y_{0} / h\right)+\sinh ^{2}\left(\pi\left(x-x_{0}\right) / h\right)}\right] .
$$

Note that when measuring $v$ along the $x(y=0)$ axis, a transverse displacement of the virtual vortex from that axis, $y_{0}$, is a higher-order effect than a streamwise displacement, $x_{0}$, i.e.

$$
\frac{v}{U_{\infty}}=\frac{a}{2 h} C_{L} \operatorname{cosech}\left(\pi \frac{x}{h}\right)+O\left(\frac{x_{0}}{h}\right)+O\left(\frac{y_{0}}{h}\right)^{2} .
$$




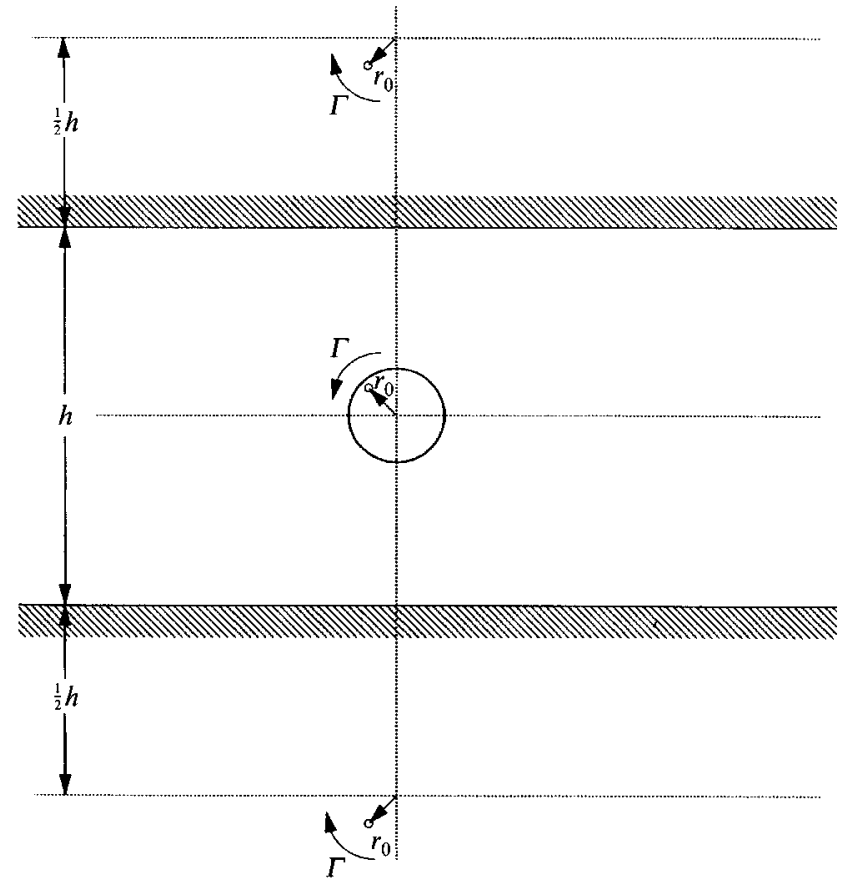

FIGURE 1. Diagram of periodic image vortices. Only the image vortices immediately above and below are pictured. The larger circle denotes the cylinder (not to scale), and the smaller circles the virtual vortex and its images.

\section{Steady rotation: $\Omega_{1}=0$}

To test the utility of $(10)-(12)$, the transverse velocity was measured at six locations ahead of the cylinder axis, for a range of $0.5 \leqslant \Omega_{0} \leqslant 10\left(\operatorname{Re}=3.8 \times 10^{3}\right)$. The validity of this model for the transverse $(v)$ velocity ahead of the cylinder is demonstrated in figure 2, a plot of $2 h v / \Gamma v s .\left(x-x_{0}\right) / a$. The parameters $h, a$, and $x$ were known $a$ priori $(h / a=40)$, and $v / U_{\infty}$ was measured. $\Gamma$ and $x_{0}$ were determined using (11) and a version of the Levenberg-Marquardt nonlinear least-squares fit routine from Press et al. (1986). Figure 3 compares the results of Reid (1924) and Prandtl (1925) with $C_{L}$ determined from this nonlinear least squares curve-fit. For these data, the cylinder aspect ratio, $A$, is defined as the ratio of the cylinder span and diameter.

It may appear that the present results measured at $R e=3.8 \times 10^{3}$ overestimate $C_{L}$ at the lowest values of $\Omega_{0}$. In fact, the results of Prandtl (1925) and Reid (1924), at $\operatorname{Re}>4 \times 10^{4}$, show a negative $C_{L}$ at the lowest values of $\Omega_{0}$. Experiments at much lower $\operatorname{Re}\left(\operatorname{Re}<100\right.$, Badr \& Dennis 1989; Tang \& Ingham 1991) yield a $C_{L}$ somewhat higher than the present experiments. Tritton (1988) reports that a sphere also experiences a lift in the 'wrong' direction at low rotation rates and higher $R e$ because of a turbulent separation on the side of the sphere moving opposite to the flow. This suggests that the discrepancy at low $\Omega_{0}$ may well be attributable to Reynolds number effects.

As can be seen from figure 3, larger cylinder aspect ratios $(A)$ yield larger maximum lift coefficients $\left(C_{L_{\max }}\right)$. Compare the results in the present experiments employing a cylinder with a larger $A=18.7$ with those of Reid $(A=13.3)$ and Prandtl $(A=4.7)$.

The curve-fit results also show that the centroid of the virtual vortex, $x_{0}$, is slightly ahead of the cylinder axis. See figure 4 . In fact, this centroid appears to be slightly upstream of the cylinder body, i.e. $x_{0} / a>1$, at the lowest value of $\Omega_{0}(=0.5)$. This may 


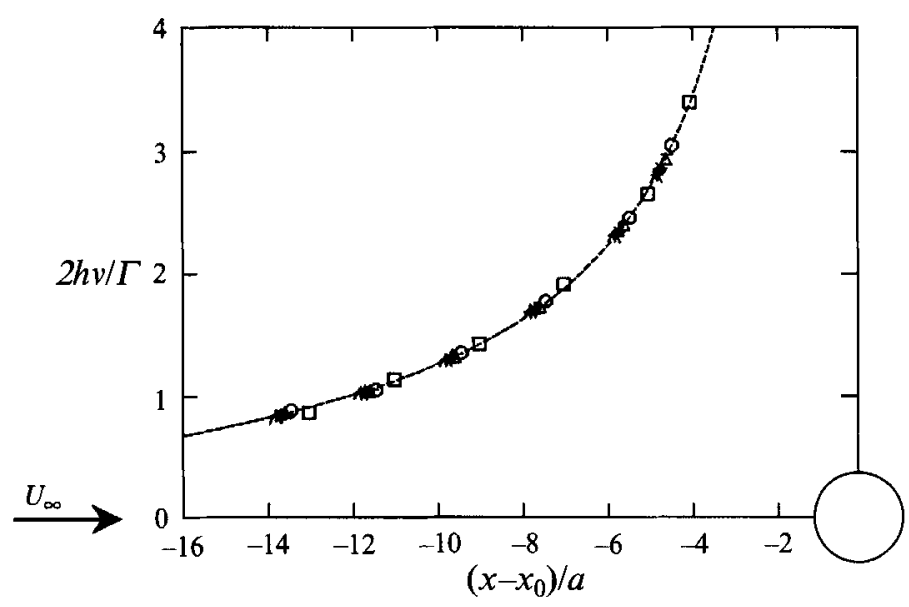

FIGURE 2. Normalized transverse velocity ahead of a cylinder executing steady rotation $\left(\Omega_{1}=0\right)$ : $2 h v / \Gamma$ vs. $\left(x-x_{0}\right) / a, R e=3.8 \times 10^{3} . \Omega_{0}=0.5(\square), 1.5(\bigcirc), 2(\triangle), 2.5(+), 3(\rightarrow), 4(\diamond), 5(*), 6(\wedge)$, $8(\mid), 10(-)$, and $\Omega_{1}=0$. The dashed line is $\operatorname{cosech}\left(\pi\left(x-x_{0}\right) / h\right)$.

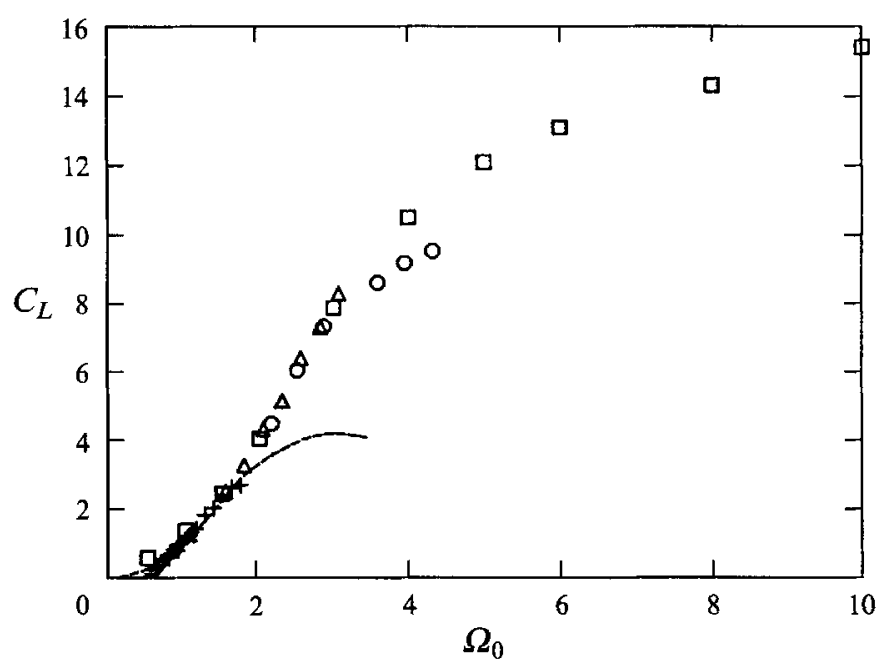

FIGURE 3. $C_{L}$ based on data fit in figure $2 R e=3.8 \times 10^{3}, \Omega_{1}=0, A=18.7$ ( $\square$ ). Data of Reid (1924) $A=13.3, R e=4.0 \times 10^{4}(O), \operatorname{Re}=5.6 \times 10^{4}(\triangle), R e=7.9 \times 10^{4}(+), R e=1.2 \times 10^{5}(\rightarrow)$. Data of Prandtl (1925) $A=4.7, R e=5.2 \times 10^{4}(--)$. $A$ is the ratio of the cylinder span and diameter.

counter intuition, as very little of the vorticity in the flow is situated upstream of the cylinder. As can be seen from figure 5 and (14), allowing for a virtual origin away from the cylinder axis is a higher-order correction.

Recall that Goldstein (1938), in an interpretation of an intuitive argument given by Prandtl (1925), suggested that $C_{L_{\text {max }}}=4 \pi \approx 12.6$. The present results, that show the estimated $C_{L}$ exceeding $4 \pi$, cast doubt on this proposed upper limit. Note that the value of $C_{L}$ measured at the highest value of $\Omega_{0}$ exceeds ' $C_{L_{\max }}$ ' by more than $20 \%$. In addition, the trend suggests that the estimated $C_{L}$ can be made larger by further increasing $\Omega_{0}$, or $A$, or both.

Diffusion and unsteady flow processes can transport vorticity away from the cylinder at start-up, weakening Prandtl's proposed $C_{L_{\max }}$ (equation (4)). A more plausible explanation is that three-dimensional (end) effects will tend to reduce the 


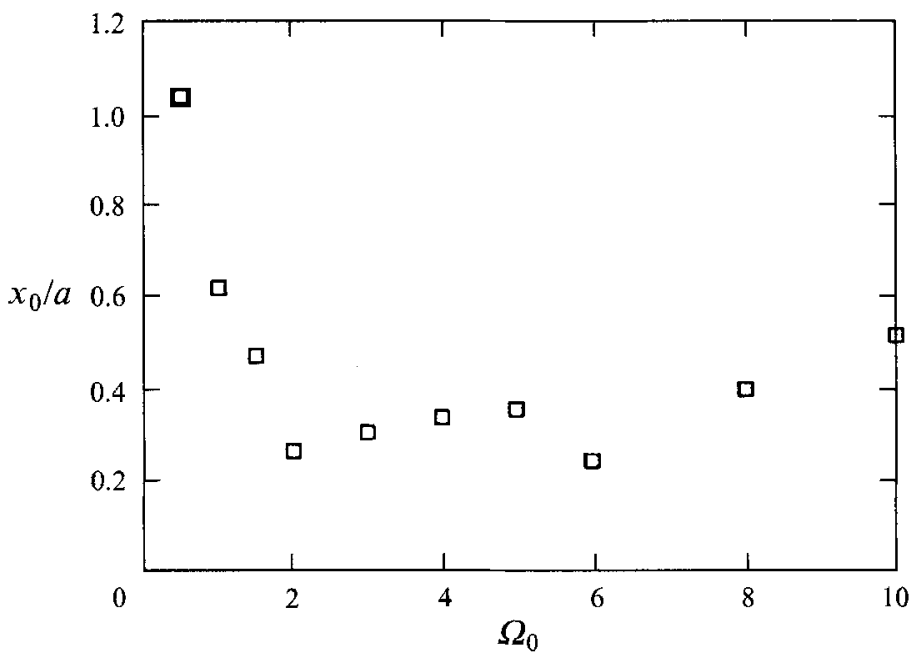

Figure 4. Centroid of the virtual vortex, $x_{0}$, based on the data fit in figure 2.

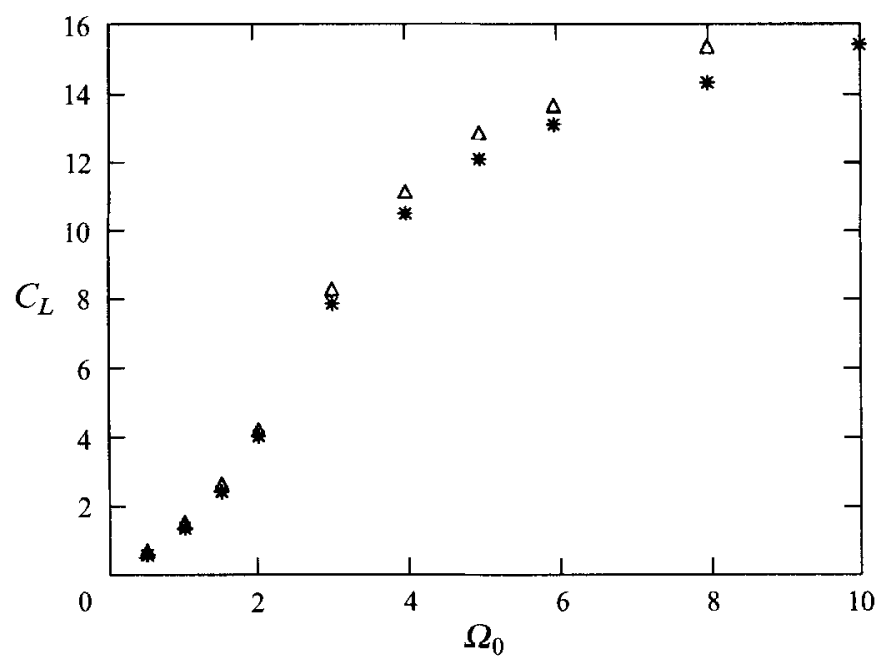

FIGURE 5. Lift estimates holding the virtual origin coincident with the cylinder axis $(\triangle)$, and allowing it to vary (*).

mean spanwise lift measured at a particular rotation rate to below that which could be attained in purely two-dimensional flow. Thom (1926) showed that the sectional lift coefficient decreased toward the ends of a rotating cylinder. It is interesting that Goldstein also relates how Prandtl, in one of his experiments, associated the limiting of $C_{L_{m a x}}(=4)$ with a separation of the flow from the sidewalls of the test section. To remedy this situation, Prandtl added co-rotating end-disks to the cylinder and then observed an increased $C_{L_{m a x}}(=10)$. No further discussion of end-effects for that configuration was included.

\section{Rotation with forced oscillations: $\Omega_{1} \neq 0, S_{f}=0.7$}

For the data presented in this section, the forcing Strouhal number, $S_{f}$, is fixed at 0.7 , while the steady and fluctuating components of the rotary motion are varied. The 


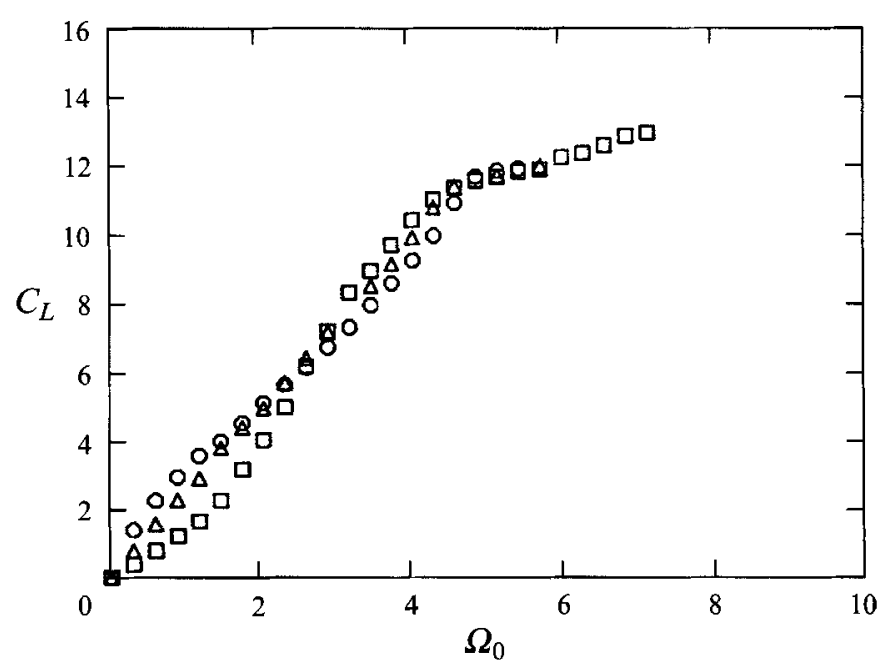

FIGURE 6. Comparison of $C_{L}$ vs. $\Omega_{0}$ data for several amplitudes of oscillations, with constant forcing frequency and Reynolds number $\left(S_{f}=0.7, R e=6.8 \times 10^{3}\right)$. $\square, \Omega_{1}=0 ; 4,1.2$; and $O, 2.3$.

Reynolds number is somewhat higher than in the previous section, at $6.8 \times 10^{3} . C_{L}$ is estimated using (12) and the mean velocity at a single point ahead of the cylinder, $v(x / a=10)$. Because $x_{0} / x \ll 1$, the centroid of the virtual vortex is taken to be on the cylinder axis, i.e. $x_{0} / h \approx 0$. See (14). In addition, the ratio of the cylinder radius to the water tunnel depth is $a / h=0.025$.

It was found that forced rotary oscillations increased $C_{L}$ for $0<\Omega_{0}<2.5$ and decreased it for $2.5<\Omega_{0}<4.5$. See figure 6. Our results and those of Badr et al. (1990) for the steadily rotating case, $\Omega_{1}=0$, show that the cylinder has noticeable periodic vortex shedding for $\Omega_{0}<2.5$, and none for $\Omega_{0}>2.5$.

Flow visualization (figures 7-9) reveals that, for $\Omega_{0}<2.5$, forced oscillations of the cylinder help close the wake, creating a flow that, on average, is closer to potential (the forced oscillations may also decrease spanwise variations in the flow). In contrast, for $2.5<\Omega_{0}<4.5$, where the wake would normally close with steady rotation alone, oscillations have the opposite effect. The data for the three cases presented in figure 6 coincide for $\Omega_{0}>4.5$, i.e. the oscillations of the cylinder have little effect on the lift beyond this point. There is also a cross-over region of the $C_{L}$ vs. $\Omega_{0}$ curve at $\Omega_{0}$ between 2 and 4 , where $C_{L}$ does not appear to be a strong function of the forcing amplitude.

\section{Streak flow visualization and streamline patterns}

For the photographs in figures 7-9, dye was introduced upstream of the cylinder near the mean 'stagnation' streamline. The overlays on the right-hand side of the figures depict the streamline patterns calculated from a potential flow model (with the cylinder bounded from above and below as in the photographs) using the lift coefficients calculated from the present experiments. As can be seen, the stagnation streakline in the photographs can be approximated by the stagnation streamline in the potential flow case. 
(a)

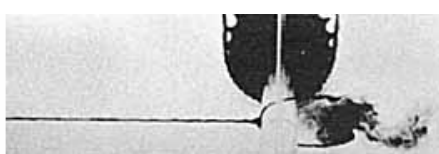

(b)

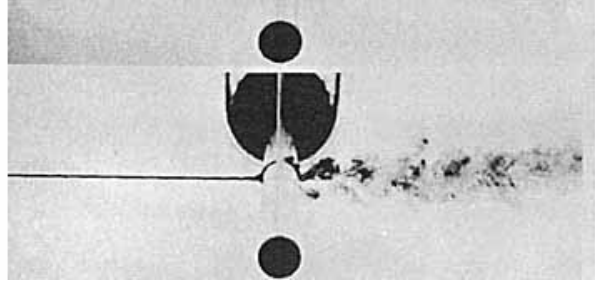

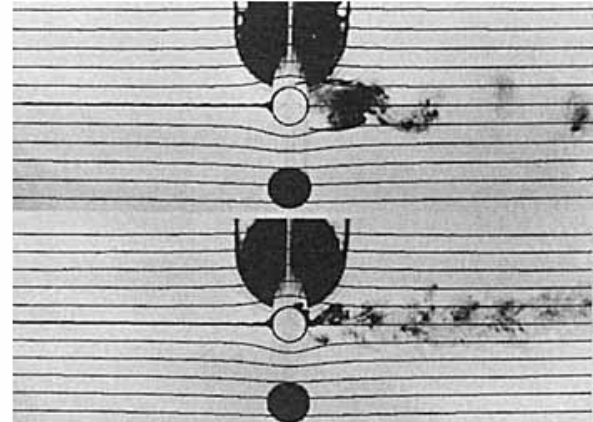

Figure 7. (a) Stationary cylinder, $\left(\Omega_{0}, \Omega_{1}, S_{f}\right)=(0,0,0), C_{L}=0$. (b) Oscillation with zero mean rotation rate, $\left(\Omega_{0}, \Omega_{1}, S_{f}\right)=(0,2.1,0.7), C_{L}=0$.

(a)

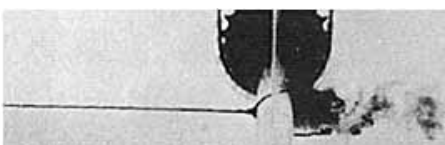

(b)

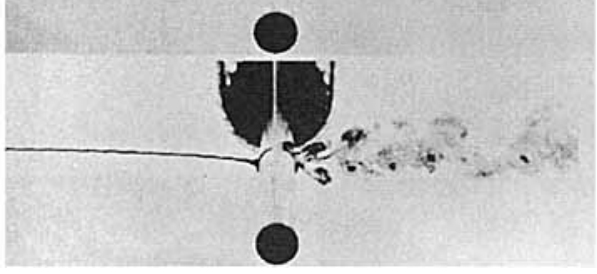

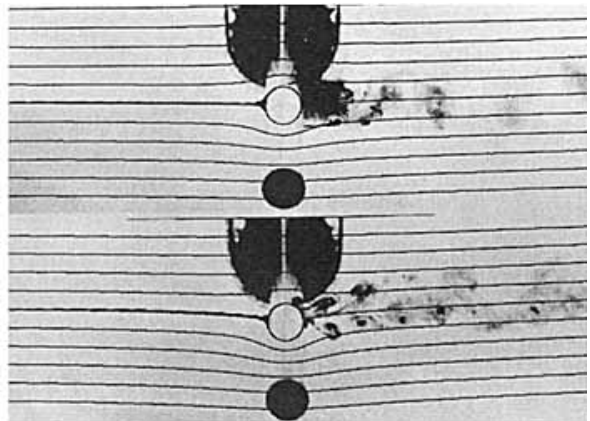

Figure 8. (a) Steady rotation, $\left(\Omega_{0}, \Omega_{1}, S_{f}\right)=(0.5,0,0), C_{L}=0.6$. (b) Oscillation with same mean rotation rate as $(a),\left(\Omega_{0}, \Omega_{1}, S_{f}\right)=(0.5,2.1,0.7), C_{L}=2.0$.

(a)

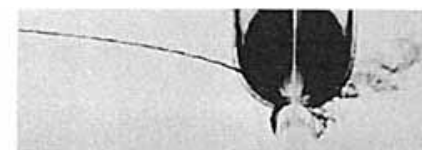

(b)

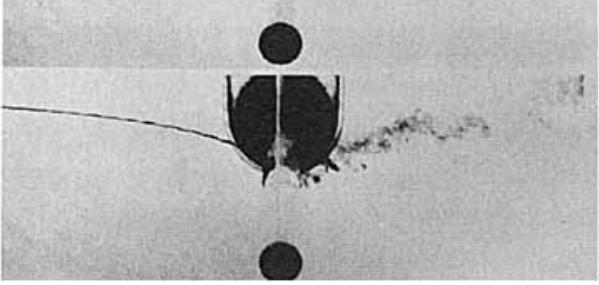

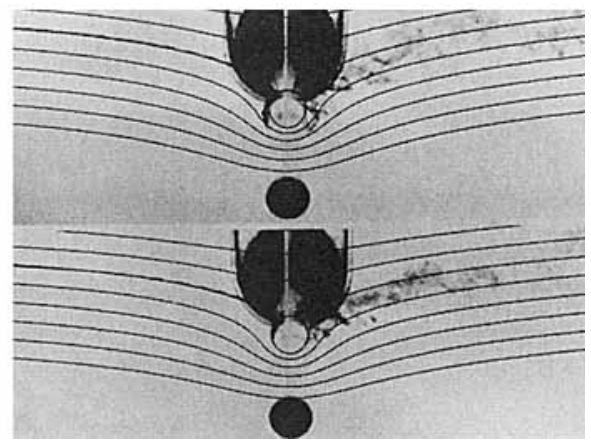

FIGURE 9. (a) Steady rotation, $\left(\Omega_{0}, \Omega_{1}, S_{f}\right)=(4,0,0), C_{L}=10.5$. (b) Oscillation with same mean rotation rate as $(a),\left(\Omega_{0}, \Omega_{1}, S_{f}\right)=(4,2.1,0.7), C_{L}=9.2$.

\section{Conclusions}

These experiments have shown that higher cylinder aspect ratios yield higher maximum lift coefficients. The maximum lift coefficients in the present experiments exceed that proposed by Prandtl (1925), possibly because Prandtl's arguments neglected unsteady effects. It was also found that addition of forced rotary oscillations to the steady rotation of the cylinder increased $C_{L}$ in cases where the wake would normally be separated (in the steadily rotating case), but decreased it otherwise. Finally, the virtual vortex method was found to be successful in characterizing the flow ahead of the cylinder. The method yielded estimates for $C_{L}$ which agree with the data 
published by Thom (1924) for lift coefficients greater than 2 , i.e. $\Omega_{0}>1$. At the smaller values of $\Omega_{0}<1$, the measurements yielded values of $C_{L}$ that are higher. This discrepancy is attributed to a difference in the Re between the experiments.

This research was sponsored by the Air Force Office of Scientific Research, URI AFOSR Grant No. F49620-86-C-0134.

\section{REFERENCES}

Badr, H. M., Coutanceau, M., Dennis, S. C. R. \& Ménard, C. 1990 Unsteady flow past a rotating circular cylinder at Reynolds numbers $10^{3}$ and $10^{4}$. J. Fluid Mech. 220, 459-484.

BADR, H. M. \& DENNIS, S. C. R. 1989 Steady and unsteady flow past a rotating circular cylinder at low Reynolds numbers. Computers Fluids 17, 579-609.

Goldstein, S. 1938 Modern Developments in Fluid Dynamics. Clarendon. (Reprinted 1965, Dover.)

INGHAM, D. B. \& TANG, T. 1990 A numerical investigation into the steady flow past a rotating circular-cylinder at low and intermediate Reynolds-numbers. J. Comput. Phys. 87, 91-107.

LANG, D. B. 1985 Laser Doppler velocity and vorticity measurements in turbulent shear layers. PhD thesis, California Institute of Technology.

MaGnus 1853 Über die Abweichung der Geschesse. Poggendorf's Annalen der Physik u. Chemie, vol. 88, pp. 1-14.

Milne-Thomson, L. M. 1968 Theoretical Hydrodynamics. Macmillan.

Prandtl, L. 1925 Die Naturwissenschaften, vol. 13, pp. 93-108. (English transl: Application of the "Magnus Effect" to the wind propulsion of ships. NACA Tech. Mem. 387, June 1926.)

Press, W. H., Flannery, B. P., Teukolsky, A. A. \& Vetterling, W. T. 1986 Numerical Recipes in $C$, The Art of Scientific Computing. Cambridge University Press.

ReID, E. G. 1924 Tests of rotating cylinders. NACA Tech. Note 209.

TANG, T. \& InghaM, D. B. 1991 On the steady flow past a rotating circular-cylinder at Reynolds numbers 60 and 100. Computers Fluids 19, 217-230.

TAYLOR, G. I. 1925 Note on the connection between the lift on an aerofoil in a wind and the circulation round it. Phil. Trans. $R$. Soc. Lond. A 225, 238-245.

THом, A. 1926 The pressure round a cylinder rotating in an air current. $A R C R . \& M .1082$.

Tном, A. 1931 Experiments on the flow past a rotating cylinder. $A R C R . \& M .1410$.

ThwaItes, B. 1960 Incompressible Aerodynamics. Clarendon. (Reprinted 1978, Dover.)

Tokumaru, P. T. 1991 Active control of the flow past a cylinder executing rotary motions. PhD thesis, California Institute of Technology.

Tokumaru, P. \& Dimotakis, P. 1991 Rotary oscillation control of a cylinder wake. J. Fluid Mech. 224, 77-90.

Trutton, D. J. 1988 Physical Fluid Dynamics, 2nd edn. Oxford University Press.

WARD, T. W. 1976 The Hydrodynamics Laboratory at the California Institute of Technology. Trans. ASME I: J. Fluids Engng 98, 740-748.

White, F. M. 1979 Fluid Mechanics. McGraw-Hill. 